\title{
EGFR and PDGFR differentially promote growth in malignant epithelioid mesothelioma of short and long term survivors
}

\author{
H Kothmaier, ${ }^{1} \mathrm{~F}$ Quehenberger, ${ }^{2}$ I Halbwedl, ${ }^{1} \mathrm{P}$ Morbini, ${ }^{3} \mathrm{~F}$ Demirag, ${ }^{4} \mathrm{H}$ Zeren, ${ }^{5}$ \\ C E Comin, ${ }^{6}$ B Murer, ${ }^{7}$ P T Cagle, ${ }^{8}$ R Attanoos, ${ }^{9}$ A R Gibbs, ${ }^{9}$ F Galateau-Salle, ${ }^{10}$ \\ H H Popper
}

- Supplementary tables 1 and 2 are published online only at http://thorax.bmi.com/content/ vol63/issue4

${ }^{1}$ Institute of Pathology, Statistics and Documentation, Medical University of Graz, Austria; ${ }^{2}$ Institute for Medical Informatics, Statistics and Documentation, Medical University of Graz, Austria ${ }^{3}$ Instituto di Anatomia Patologica, IRCCS Policlinico S Matteo, Pavia, Italy:

${ }^{4}$ Department of Pathology, Atatürk Chest Disease and Chest Surgery Education and Research Hospital, Ankara, Turkey: ${ }^{5}$ Pathology, Adana University, Adana, Turkey; ${ }^{6}$ Department of Human Pathology and Oncology, University of Florence, Italy;

${ }^{7}$ Department of Anatomic

Pathology, Mestre, Italy;

${ }^{8}$ Department of Pathology, The Methodist Hospital and Cornell University Houston, Houston, Texas, USA; ${ }^{9}$ Department of Histopathology, Cardiff and Vale NHS Trust, Llandough Hospital, Penarth Vale of Glamorgan, Cardiff, UK: ${ }^{10}$ Laboratoire d'Anatomie Pathologique, $\mathrm{CHU}$ Cote de Nacre, Caen Cedex, France

Correspondence to: Dr H H Popper, Institute of Pathology, Medical University of Graz, Auenbruggerplatz 25 A-8036 Graz, Austria; helmut.popper@ meduni-graz.at

Received 11 June 2007 Accepted 4 October 2007 Published Online First 17 December 2007

\section{ABSTRACT}

Background: Malignant pleural mesothelioma (MPM) is an asbestos related tumour difficult to detect early and treat effectively. Asbestos causes genetic modifications and cell signalling events that favour the resistance of MPM to apoptosis and chemotherapy. Only a small number of patients, approximately 10\%, survive more than 3 years. The aim of our study was to assess possible differences within signalling pathways between short term survivors (survival $<3$ years; STS) and long term survivors (survival $>3$ years; LTS) of MPM.

Methods: 37 antibodies detecting proteins engaged in cell signalling pathways, enforcing proliferation, antiapoptosis, angiogenesis and other cellular activities were investigated by tissue microarray (TMA) technology. Results: Epidermal growth factor receptor (EGFR) was expressed stronger in LTS whereas platelet derived growth factor receptor (PDGFR) signalling was more abundant in STS. Expression of TIE2/Tek, a receptor for tyrosine kinases involved in angiogenesis, was differentially regulated via PDGFR and thus is more important in STS. Antiapoptosis was upregulated in STS by signal transducer and activator of transcription 1 (STAT1)survivin and related molecules, but not in LTS. Our study provides novel insights into the regulatory mechanisms of signalling pathways in MPM, which differentially promote tumour growth in LTS and STS.

Conclusion: We have demonstrated that small scale proteomics can be carried out by powerful linkage of TMA, immunohistochemistry and statistical methods to identify proteins which might be relevant targets for therapeutic intervention.

Malignant pleural mesothelioma (MPM) is an asbestos related tumour difficult to detect early and treat effectively. ${ }^{1}$ Although MPM in general is a rare tumour, its incidence is increasing worldwide. It is estimated that in Europe, Australia and Japan, MPM will peak between 2015 and 2025, whereas in the USA, MPM reached its peak incidence in $2004 .^{2}$

Most cases are a direct consequence of asbestos exposure 30-40 years earlier. From the onset of symptoms, survival is a few weeks to a few years, ${ }^{3}$ median survival being 4-12 months in treated or untreated patients. ${ }^{4}$ Only a small number of patients (approximately 10\%) survive more than 3 years, which seems to be restricted to the epithelioid subtype. ${ }^{5}$ Immunohistochemical analysis of Ki-67/MIB-1,7 p27 ${ }^{\mathrm{kip} 1,8}$ p21 ${ }^{\mathrm{cip} 1}$ and cyclooxygenase- $2^{59}$ have been correlated with survival in
MPM, but their significance has not been entirely clarified.

Asbestos causes genetic modifications and cell signalling events, most notably the upregulation of cell survival and growth pathways, as well as other proteins that favour the resistance of MPM to apoptosis and chemotherapy. ${ }^{10}$ Various studies have confirmed the importance of signalling pathways in MPM, including the epidermal growth factor (EGF), platelet derived growth factor (PDGF), hepatocyte growth factor and insulin-like growth factor (IGF) pathways and their downstream signalling molecules, such as the mitogen activating protein kinase and phosphatidylinositol 3-kinase (PI3-K)/Akt kinase, respectively. ${ }^{11} 12$

The aim of our study was to compare the role of signalling pathways between short term survivors $(<3$ years; STS) and long term survivors (>3 years; LTS) of MPM, assessing possible differences correlated to the corresponding phenotype. To do this, a large panel of antibodies detecting proteins engaged in these cell signalling pathways, enforcing proliferation, antiapoptosis, angiogenesis and other cellular activities in MPM were investigated by tissue microarray (TMA) technology. Because of the extreme rarity of LTS in MPM, a comparison between STS and LTS with regard to signalling pathways has never been investigated previously.

\section{METHODS}

\section{Histological examination and clinical data}

Seventy MPM were derived from surgically resected material, either open biopsies or pleurectomies. The cases were diagnosed between 1987 and 2003 and classification was made according to the World Health Organisation (WHO 2004) by each of the contributing authors (PM, FD, HZ, CEC, BM, PTC, RA, ARG., FGS, HHP). At least three positive and two negative markers were applied to confirm the diagnosis of a mesothelioma according to published recommendations. ${ }^{13}$ Fortyeight of 70 cases of epithelioid MPM (collected in USA, UK, Turkey, Italy, France and Austria) with available clinical information and sufficient formalin fixed, paraffin embedded material were selected for TMA construction. Asbestos exposure was confirmed in one-third of the patients in both groups; in the others no exposure data were available. Informed consent from patients was provided with the data sheet submitted with the 
Table 1 Clinical data for the 48 patients with epithelioid malignant pleural mesothelioma involved in the study

\begin{tabular}{llllll}
\hline $\begin{array}{l}\text { MPM } \\
(\mathbf{n}=\mathbf{4 8})\end{array}$ & Males & Females & $\begin{array}{l}\text { Age (y) } \\
\text { (range (mean)) }\end{array}$ & $\begin{array}{l}\text { Survival (months) } \\
\text { (range (mean)) }\end{array}$ & MIB1/Ki-67 \\
\hline LTS $(\mathrm{n}=26)$ & 11 & 15 & $41-78(63.7)$ & $36-116(50.7)$ & $\begin{array}{l}\text { Cases }<30 \% \\
24 / 26(92.3 \%) \\
\text { Cases }>30 \%\end{array}$ \\
STS $(\mathrm{n}=22)$ & 17 & 5 & $30-71(57.4)$ & $<1-31(9.1)$ & $13 / 22(40.9 \%)$
\end{tabular}

36 months was taken as the cut-off level for survival; 26 patients were identified as LTS and 22 as STS.

MIB-1/Ki-67 proliferation index $(<30 \%$ or $>30 \%$ ) was associated with STS and LTS.

LTS, long term survivors; MPM, malignant pleural mesothelioma; STS, short term survivors.

tissue. In addition, the study was approved by the local Ethical Commission.

Taking 36 months as a cut-off level for survival, 26 patients were identified as LTS and 22 as STS. Survival time was determined from the time of diagnosis until the last follow-up or death. Fifteen of 20 females and 11 of 28 males were LTS. Clinical data were collected for all patients (table 1).

\section{Tissue microarray construction}

For TMA construction, a haematoxylin-eosin (H\&E) stained section was cut from each paraffin block and re-examined by an experienced pathologist (HP). Five representative tumour regions were morphologically identified and marked on the $\mathrm{H} \& \mathrm{E}$ stained sections. Tissue cylinders with a diameter of $0.6 \mathrm{~mm}$ were punched from the marked areas of each block and brought into a recipient paraffin block, using a manual instrument (Beecher Instruments, Sun Prairie, Wisconsin, USA). Sections (5 $\mu \mathrm{m}$ thick) were cut from each TMA and prepared for immunohistochemistry as previously described. ${ }^{14}$ Every 15 th section was stained with $H \& E$ and controlled for the presence of the epithelioid subtype. To overcome the problem of tumour heterogeneity and increase the number of accessible slides, each donor tissue block was punched 6-10 times for construction of two recipient blocks, each containing 243 tissue cores. In these 243 tissue cores, 13 adjacent parenchyma cores were also included.

\section{Immunohistochemistry}

Immunohistochemical staining with 37 antibodies was performed on TMA sections, according to the manufacturer's recommendations. In addition, antibodies were pretested on different tissues prior to their use on TMA. Antibodies used in the study for simplicity of reading are given in the abbreviated form (in alphabetically order): AMPK $\alpha 2, \beta$-catenin, CREB binding protein, c-Fos, c-Jun, c-Met, c-Myc, cyclin D1, epidermal growth factor receptor (EGFR)1, extracellular signal regulated kinase 2 (ERK2), Gab1, Grb2, glycogen synthase kinase 3 (GSK3), IGF1R, IGF1R $\alpha$, Janus kinase 1 (JAK1), MAP4K-1, methyl-CpG binding protein 2 (MECP2), p-m-Tor (Ser2448), NFkB p65, pAKT (Ser473), proliferating cell nuclear antigen (PCNA), Src, p27Kip1, platelet derived growth factor receptor (PDGFR) $\alpha$, PDGFR $\beta$, PI3K $p 110 \alpha$, p-ELK, p-p70S6K(Ser411), p-Paxillin, signal transducer and transcription factor (STAT)1, STAT3, STAT5, survivin, transforming growth factor (TGF) $\beta$, TIE2 and VEGF. A list of the antibodies with additional information about source, dilution, antigen retrieval and detection is given in supplementary table 1 (available online).

\section{Scoring}

Immunohistochemical analysis was carried out by one pathologist (HP) without knowledge of patient survival data or core distribution within the TMA. Protein expression was recorded semiquantitatively. For each core, staining intensity ( 0 , no staining; $1+$, weak; $2+$, moderate; $3+$, strong) together with the percentage (0-100\%, in 10\% increments) of cells expressing the protein was recorded. Staining scores were calculated by multiplying the percentage of positive cells by the staining intensity. The obtained product scores, ranging from 0 to 300 , were used for statistical analysis.

\section{Statistics}

The null hypothesis that there is no difference in immunohistochemical parameters between LTS and STS was assessed with Goeman's global test, the R-package globaltest 3.0.2 (http:// www.bioconductor.org/repository/release1.5/package/html/ globaltest.html) with a simulated permutation test criterion. Individual immunohistochemical parameters were tested for differences using Wilcoxon's rank sums test. Correlation coefficients were calculated using Spearman's rank correlation coefficient of the product score. Tests of differences between correlation coefficients of LTS and STS were based on differences between $\mathrm{z}$ transformed Spearman correlation coefficients. $p$ Values were calculated from simulated permutation tests.

The statistically relevant correlations and differences between correlations were displayed as network graphics with antibodies as nodes and correlation as edges (see fig 3). p Values below 0.05 were considered to be statistically significant. All permutation tests comprised 10000 replications.

Correlations between immunohistochemical parameters were displayed as network graphic using Graphviz graph visualisation software (http://www.graphviz.org). The absolute values of correlations determined the strength of springs between nodes. The neato algorithm placed the nodes such that the energy of the system was minimised.

\section{RESULTS}

\section{Clinical features}

Forty-eight MPM cases used for the TMA construction were histologically classified as epithelioid mesotheliomas. Survival of the 26 LTS (survival >36 months) ranged from 36 to 116 months, with a mean of 50.7 months, whereas the 22 STS ( $<36$ months) ranged from $<1$ to 31 months with a mean of 9.4 months. The mean survival time in the LTS group was 5.4 times longer than that in the STS group (table 1). Sex was neither significantly correlated to survival nor to immunohistochemical reactions.

\section{Immunohistochemistry and statistical evaluation}

MIB-1 immunostaining revealed diffuse nuclear staining. In line with published data, the proliferative activity, detected by MIB-1 


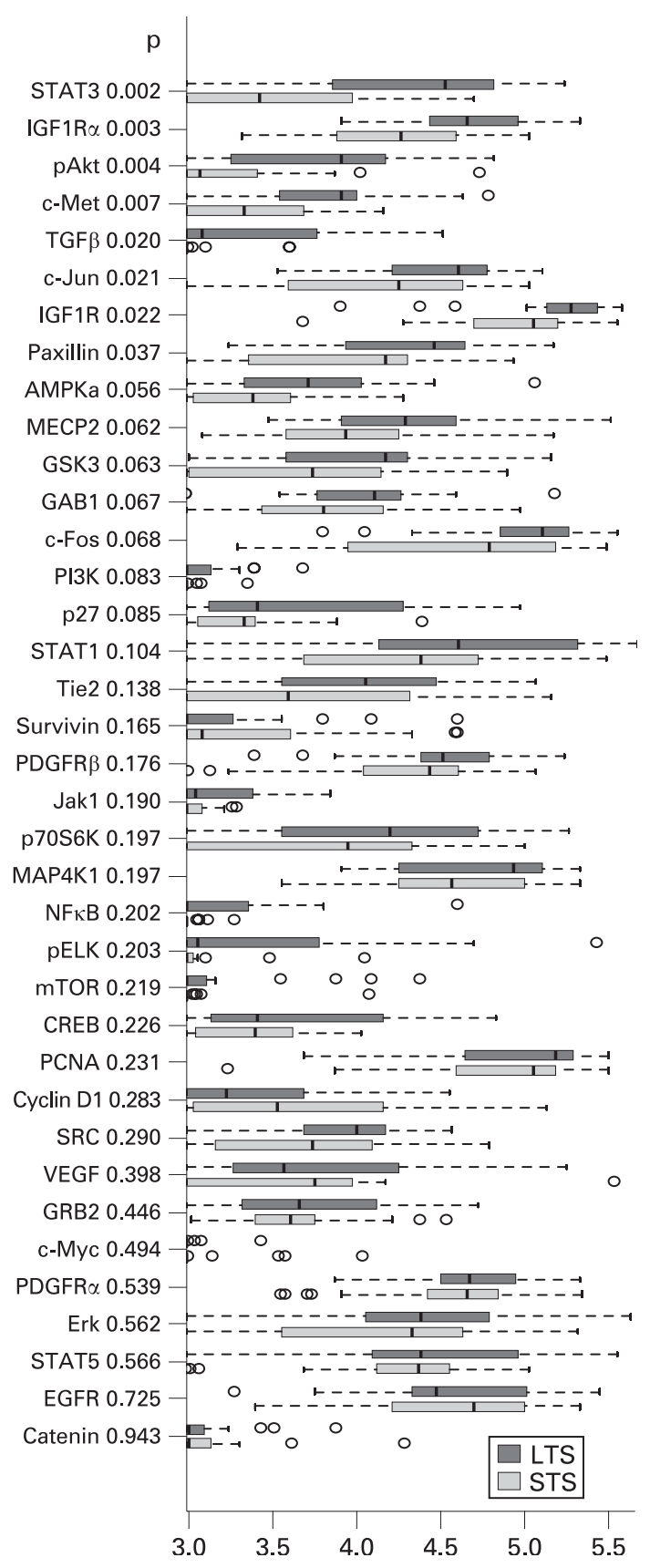

Figure 1 Box and whisker plots of all antibody measurements for long term survivors (LTS) and short term survivors (STS). The log2 $(x+20)$ transformed product score is shown on the horizontal axis. On the vertical axis for each antibody is the $p$ value of the Wilcoxon rank sum test which compares the median of the product score in LTS and STS. In LTS, significantly higher values $(p<0.05)$ were found for STAT3, IGF1Ra, pAKT, c-Met, TGF $\beta$, c-Jun, IGF1R and paxillin.

immunoreactivity, was significantly different between LTS and STS ( $p=0.014$, Fisher's exact test) (table 1 ).

Many of the proteins analysed on the TMA were characterised by low expression in the parenchyma and increased expression in the tumour tissue. Comparison of protein expression profiles between LTS and STS showed a significant difference ( $p<0.009$, Goeman's global test).

In the comparison of expression levels of single proteins detected by the corresponding antibodies, significantly higher values were found in LTS only for STAT3, IGF1Ra, pAKT,
c-Met, TGF $\beta$, c-Jun, IGF1R and paxillin ( $p<0.05$, Wilcoxon's rank sum test) (fig 1).

Representative immunohistochemical stains for EGFR, PDGFR, TIE2, STAT1, STAT3 and survivin are shown in fig $2 \mathrm{~A}-\mathrm{F}$.

Correlations and correlation differences between LTS and STS are displayed in the network graphic (fig 3) and the corresponding correlation differences are summarised in table 2. Differences in correlations indicate differences in the underlying regulatory network. ${ }^{15}$ The complete statistical data set, including correlation and $p$ values, is given for LTS and STS and the differences in correlations between LTS and STS ( $z$ transformed correlations) in supplementary table 2A-C (available online).

\section{DISCUSSION}

We have explored differences in signalling pathways by defining correlation differences in protein expression between LTS and STS (table 2). Only those differences for which a rationale to signalling pathways exist are included in the discussion below. These differences indicate alterations in the underlying regulatory pathway, including proliferation (EGFR or PDGFR), angiogenesis (TIE2) and antiapoptosis (STAT1, survivin), which seem differentially regulated in both groups. A simplified schema showing interactions of EGFR and PDGFR signalling is given in fig 4.

\section{EGFR signalling in LTS}

In the LTS, EGFR is correlated with downstream acting STAT3, the proto-oncogenes c-Fos and c-Jun, and proliferating cell nuclear antigen (PCNA). Further EGFR downstream acting proteins including GAB1, Janus kinase 1 (JAK1, plays a pivotal role in phosphorylating STAT3), extracellular signal regulated kinase 2 (ERK2), pAKT and pELK are highly correlated in LTS but not detected in STS. These results indicate that cell survival pathways in LTS are preferentially driven through EGFR. ERK, Akt and STAT3 are on signal transduction pathways triggered by EGFR ${ }^{16}$ and the subsequent activation of c-Fos, c-Jun and PCNA is caused by EGFR activation also. ${ }^{17}$ Blocking EGFR signalling with the EGFR inhibitors gefitinib and erlotinib in early phase II trials of patients with MPM had limited or no effect, ${ }^{18}$ suggesting that EGFR is a therapeutic target only for a minority of mesothelioma patients. ${ }^{16}$ From our findings we suppose that this minority belongs to the LTS.

Our data demonstrate that TGF $\beta$ promotes EGFR signalling in the LTS. It is known that TGF $\beta$ activates EGFR by inducing expression of the EGFR ligands TGF $\alpha$ and heparin binding EGFlike growth factor. ${ }^{19}$ The ligands form dimers and after receptor binding EGFR will be activated for further downstream signalling. TGF $\beta$ might be responsible for EGFR activation in LTS because of the higher expression value in LTS $(p=0.02$, fig 1) compared with STS as well as TGF $\beta$ which is correlated with PCNA and IGF1R. The latter is also more highly expressed in LTS ( $p=0.003$, fig 1$)$.

\section{PDGFR signalling in STS}

Non-neoplastic mesothelial cells express predominantly the $\operatorname{PDGFR} \alpha$ subunit and less PDGFR $\beta$, while MPM prefers PDGFR $\beta$ expression. ${ }^{20}$ In our cases both PDGFR $\alpha$ and PDGFR $\beta$ were expressed equally in LTS and STS. PDGFR $\alpha$ expression in MPM could be induced by interferon gamma (IFN $\gamma$ ). In THP1 cells it was shown that IFN $\gamma$ stimulation resulted in augmented expression of PDGFR $\alpha$ through transient STAT1 promoter 
Figure 2 Immunohistochemical stains. Immunohistochemical stainings for $(A)$ epidermal growth factor receptor (EGFR), (B) platelet derived growth factor receptor (PDGFR), (C) TIE2, (D) signal transducer and activator of transcription (STAT)1, (E) STAT3 and (F) survivin of different cores from the epithelioid malignant pleural mesothelioma tissue microarray (original magnification $\times 200$ ). Small bars represent $20 \mu \mathrm{m}$, large bars $50 \mu \mathrm{m}$.

A
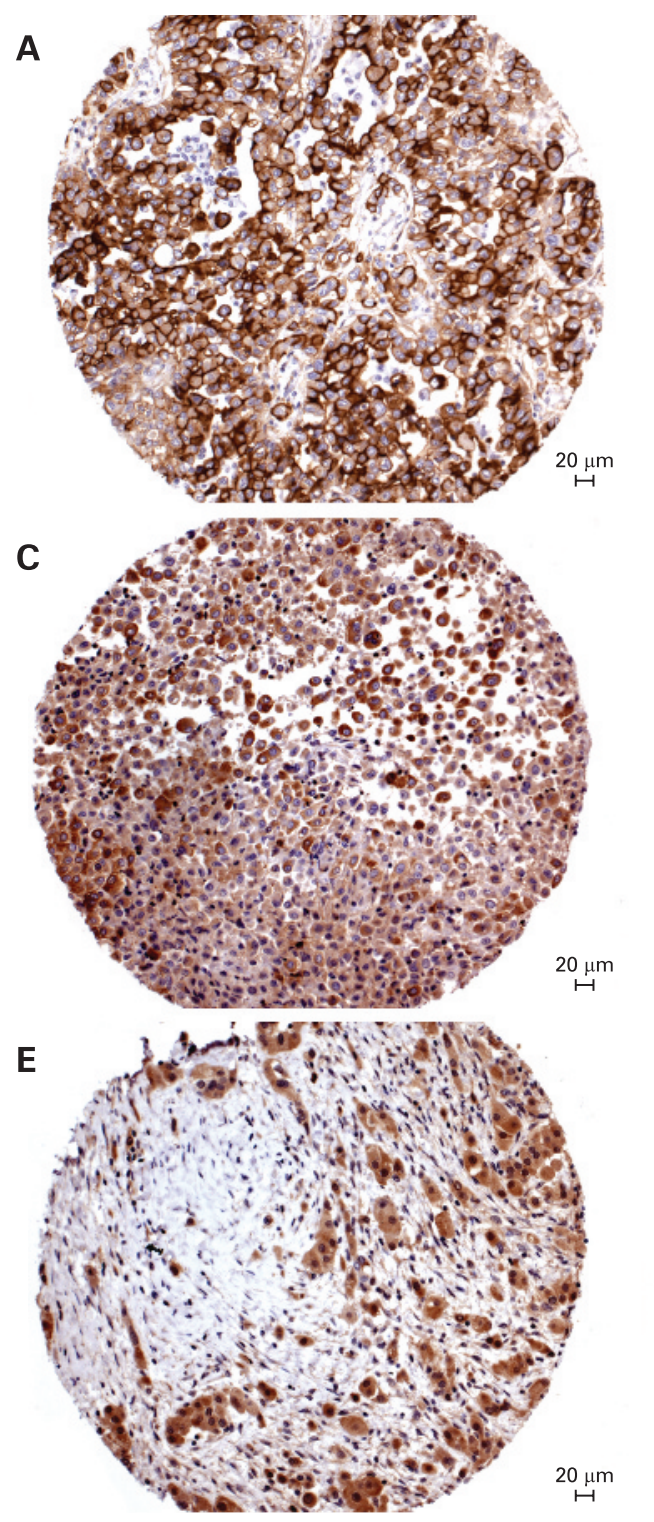

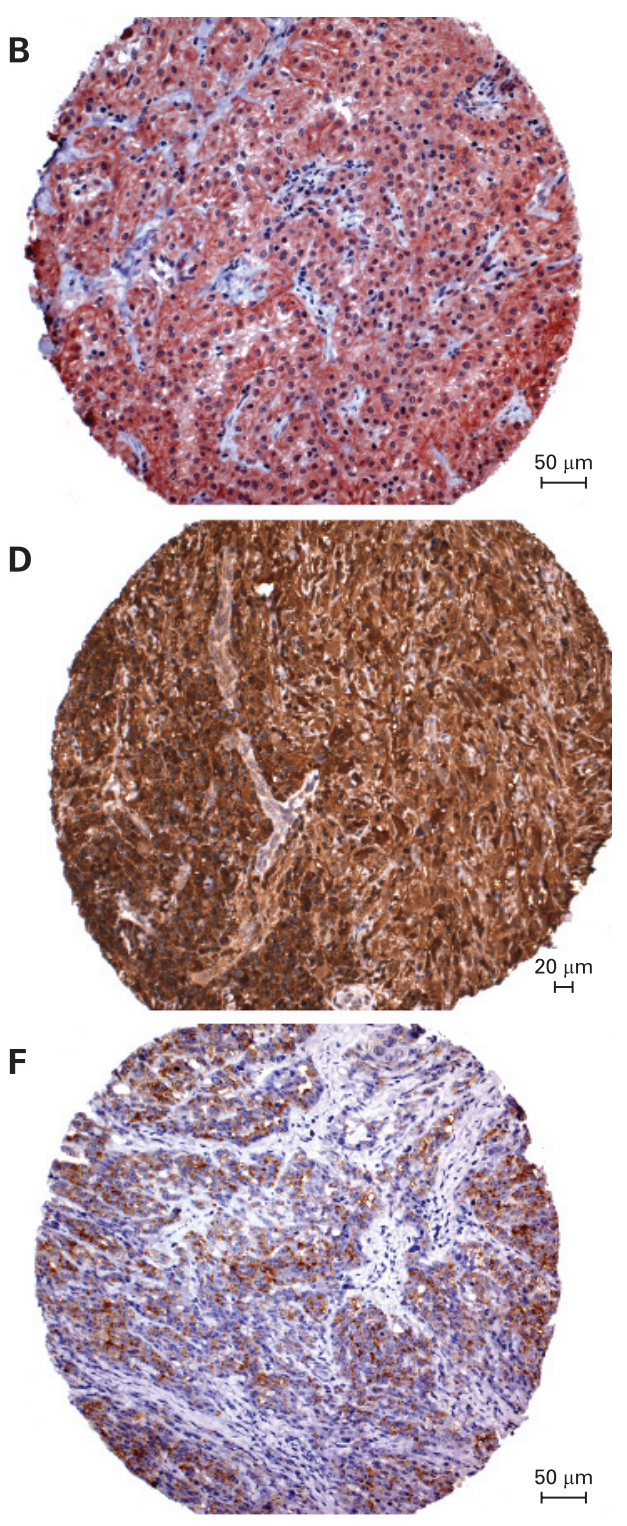

binding, ${ }^{21}$ and IFN $\gamma$ was described as inducing STAT1 overexpression. ${ }^{22}$

Only in STS did we found PDGFR $\alpha$ and PDGFR $\beta$ associated with downstream glycogen synthase kinase 3 (GSK3), TIE2, c-Fos, cyclin D1 and PCNA. Some of these downstream acting proteins (TIE2, c-fos and PCNA) were also important in LTS. But in LTS these three proteins were associated with EGFR expression. Analysis of correlation differences indicates that PDGFR and EGFR pathways were differentially activated in both groups. Although STS and LTS show similar expression levels of EGFR and PDGFR, correlation analysis showed that LTS prefer EGFR whereas STS prefer PDGFR signalling pathways with different downstream proteins.

PCNA could be a key player in MPM proliferation in both STS and LTS. Interestingly, it is known that binding of EGF, PDGF and IGF1 to their corresponding receptors induces PCNA expression and stabilisation of the resulting mRNA. ${ }^{23}$ Taking this into account, our results imply that PCNA expression benefits from EGFR and/or PDGFR activation in LTS and STS. Blocking key proteins acting within several signalling pathways might be potential targets for further translational approaches in MPM. PCNA alone or in combination with PDGFR could be one of these targets.

\section{TIE2 and angiogenesis in MPM}

TIE2/Tek, a recently identified RTK principally expressed on vascular endothelium, induces angiogenesis on stimulation through one of the four angiopoetin ligands (Ang1-4). To date, TIE2 protein expression has not been studied in MPM but TIE2 involvement has been reported for breast, ${ }^{24}$ lung $^{25}$ and liver tumours. ${ }^{26}$ In STS we found TIE2 highly associated with PDGFR $\alpha$ and PDGFR $\beta$. In contrast with LTS, TIE2 was associated with EGFR. Hence we conclude that PDGFR expression in STS and EGFR in LTS leads to a subsequent upregulation of TIE2 in MPM.

\section{Antiapoptosis induced by survivin and STAT1 in STS}

Survivin, a member of the inhibitor of apoptosis protein family, is specifically upregulated in a variety of human cancers and undetectable in normal tissue. ${ }^{27}$

In our cases, survivin was also exclusively expressed in the tumour tissue whereas it was not detected in adjacent normal pleura. Lower expression of survivin was found in LTS compared with STS (fig 1). Xia and colleagues ${ }^{27}$ previously reported that overexpression of survivin in mesothelioma cell 
Figure 3 Network graphic. The network graphic shows the correlations between antibodies using the Spearman rank correlation of the product score. There are 666 possible correlations between the antibodies but only correlations higher than 0.7 are shown. Blue edges indicate correlations within long term survivors (LTS), red edges indicate correlations within short term survivors (STS). Black edges represent differences in correlations between LTS and STS with a $\mathrm{p}$ value below 0.05 . Nodes represent the proteins detected by the antibody. The neato algorithm placed correlated antibodies close to each other.

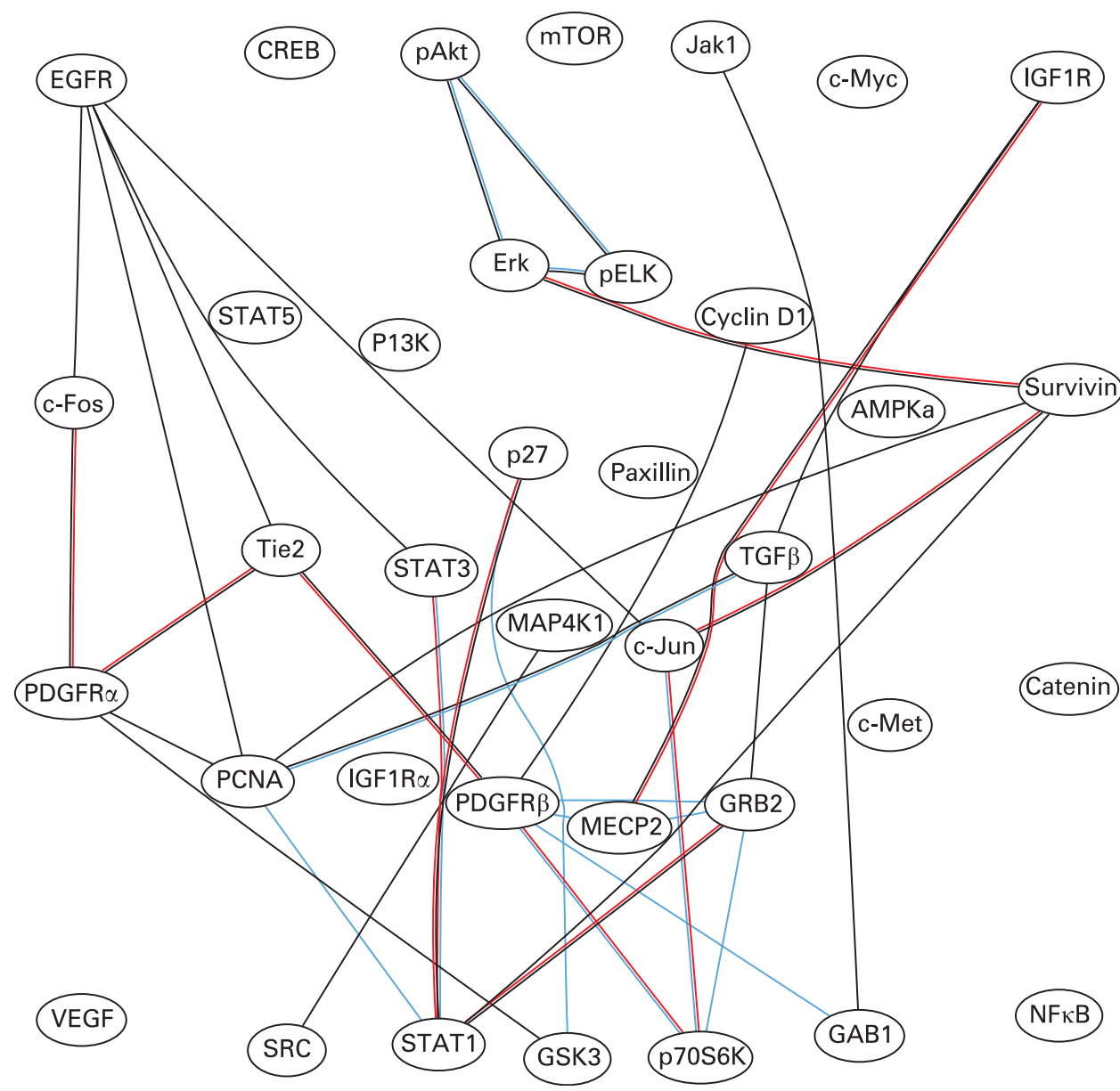

lines and fresh tumour samples contribute to the poor response of MPM cells to chemo- and radiation therapy.

Survivin in STS is highly correlated with ERK2, STAT1, c-Jun and PCNA. The correlation between survivin and STAT1 indicates a novel role for STAT1 promoting antiapoptosis through survivin upregulation. For chemotherapy resistant breast carcinomas it was reported that survivin was induced by STAT3. In addition, the authors showed that direct inhibition of STAT3 signalling blocked survivin expression. ${ }^{28}$ Contrary to breast carcinomas, our findings provide a strong

Table 2 Differentially correlated proteins in STS and LTS with $p<0.05$

\begin{tabular}{|c|c|c|c|c|c|c|c|}
\hline \multicolumn{3}{|c|}{ Higher correlations in STS } & \multirow[b]{2}{*}{ p Value } & \multicolumn{3}{|c|}{ Higher correlations in LTS } & \multirow[b]{2}{*}{ p Value } \\
\hline Protein 1 & Protein 2 & $\begin{array}{l}\text { Correlation } \\
\text { difference }\end{array}$ & & Protein 1 & Protein 2 & $\begin{array}{l}\text { Correlation } \\
\text { difference }\end{array}$ & \\
\hline $\operatorname{PDGFR} \alpha$ & Tie2 & 0.77 & 0.036 & EGFR & PCNA & 1.01 & 0.004 \\
\hline PDGFR $\beta$ & Tie2 & 0.78 & 0.045 & EGFR & STAT3 & 0.80 & 0.027 \\
\hline $\operatorname{PDGFR} \alpha$ & GSK3 & 0.73 & 0.039 & EGFR & C-Jun & 0.70 & 0.038 \\
\hline $\operatorname{PDGFR} \alpha$ & c-Fos & 0.73 & 0.035 & EGFR & Tie2 & 0.86 & 0.013 \\
\hline $\operatorname{PDGFR} \alpha$ & PCNA & 0.70 & 0.045 & EGFR & c-Fos & 0.65 & 0.046 \\
\hline PDGFR $\beta$ & Cyclin D1 & 0.67 & 0.049 & Erk & pAkt & 0.84 & 0.027 \\
\hline STAT1 & p27 & 0.96 & 0.013 & pELK & pAkt & 0.82 & 0.047 \\
\hline STAT1 & Survivin & 0.76 & 0.025 & Erk & pELK & 0.81 & 0.028 \\
\hline STAT1 & GRB2 & 0.77 & 0.029 & GAB1 & Jak1 & 0.67 & 0.047 \\
\hline Survivin & Erk & 0.91 & 0.012 & IGF1R & TGF $\beta$ & 0.87 & 0.005 \\
\hline Survivin & C-Jun & 0.85 & 0.022 & PCNA & TGF $\beta$ & 0.87 & 0.007 \\
\hline Survivin & PCNA & 0.73 & 0.032 & & & & \\
\hline IGF1R & MECP2 & 0.87 & 0.017 & & & & \\
\hline MAP4K1 & SRC & 0.69 & 0.042 & & & & \\
\hline
\end{tabular}

The resulting differences between correlations are given either in the left or right section of the table, depending on whether correlations were higher in STS or LTS, respectively.

EGFR, epidermal growth factor receptor; GSK3, glycogen synthase kinase 3; IGF1R, insulin-like growth factor receptor 1; LTS, long term survivors; MECP2, methyl-CpG binding protein 2; PCNA, proliferating cell nuclear antigen; PDGFR, platelet derived growth factor receptor; STAT, signal transducer and activator of transcription; STS, short term survivors; TGF $\beta$, transforming growth factor $\beta$. 
Figure 4 Schema showing the different interactions of epidermal growth factor receptor (EGFR) and platelet derived growth factor receptor (PDGFR) signalling with respect to long term (LTS) and short term (STS) survivors in malignant pleural mesothelioma (MPM). All molecules shaded in grey have been tested on the tissue microarray and molecules not tested in white; arrows indicate activation, $\mathrm{P}$ indicates phosphorylation. For simplicity, only major key proteins are included.

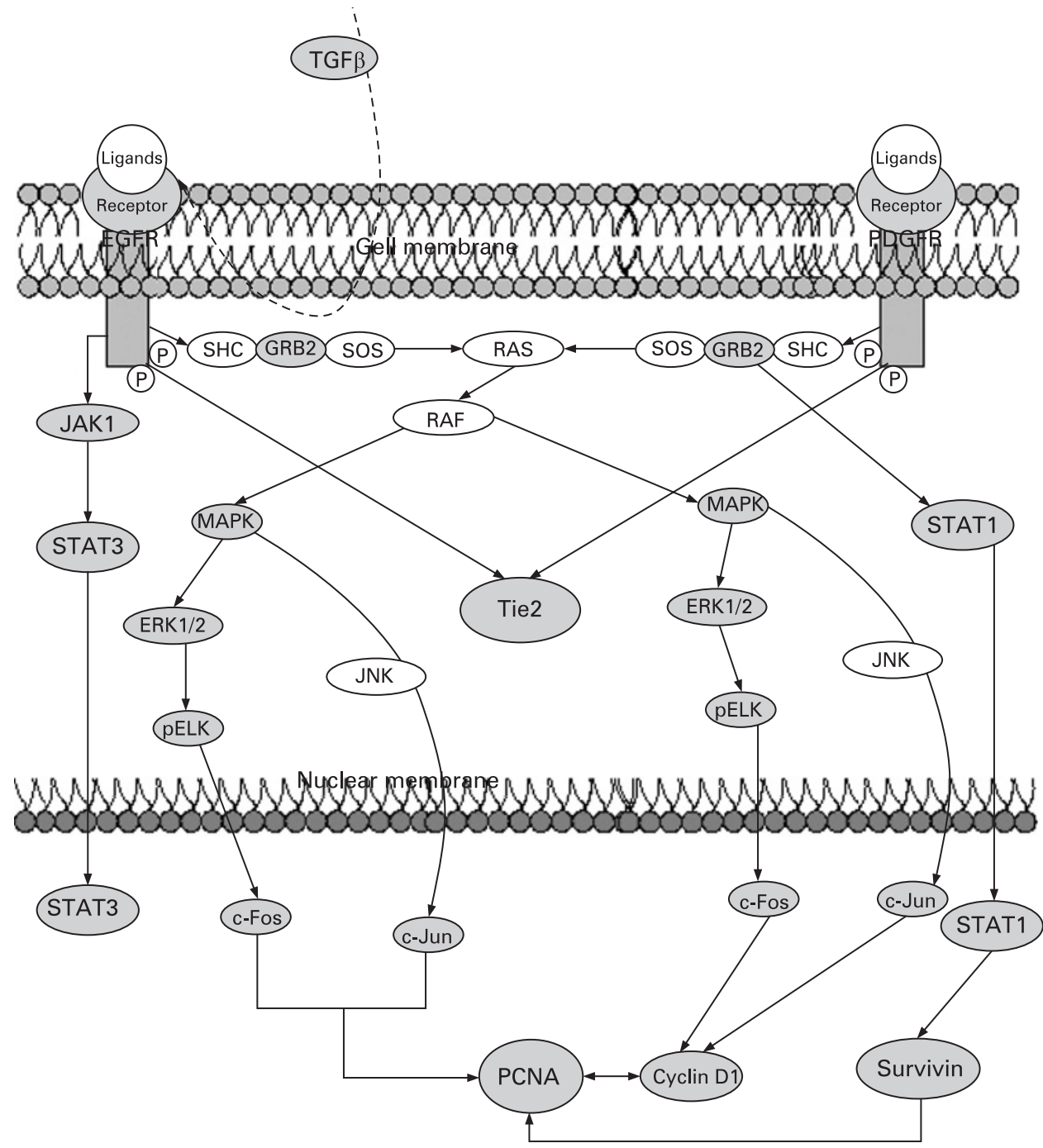

argument that in STS of MPM, survivin upregulation is induced by STAT1, instead of STAT3. Survivin disrupts the intrinsic apoptosis pathway, most probably by blocking activation of caspase 9.29 Enhanced STAT1-survivin and PCNA-survivin interactions imply that in STS, proliferation is promoted and apoptosis is blocked via survivin expression, while in LTS survivin plays no significant role.

\section{STAT1 and MECP2, a novel role in MPM pathogenesis}

STATs are a family of latent cytoplasmic signal transducers and activators of transcription with known opposing actions. While STAT3 and STAT5 are referred to as the oncogenic STATs, STAT1 is regarded as a tumour suppressor. ${ }^{30}$ Curiously, we found STAT1 and STAT5 equally expressed in LTS and STS, whereas STAT3 was higher expressed in LTS ( $p=0.03$, fig 1$)$. In LTS, we found STAT3 further associated with EGFR.

It appears that in MPM the tumour suppressor STAT1 acts similar to an oncogene. Exclusively in STS, STAT1 was correlated with survivin, p27 and Grb2. STAT1 overexpression has also been reported in breast, head and neck cancers, and in some haematological tumours, again suggesting a prosurvival mode of action, ${ }^{31}$ but its role has not been clarified. Only one report describes the role of STAT1 in mesothelioma. ${ }^{22}$ Buard et al reported that IFN $\gamma$ induces STAT1 overexpression in mesothelioma cell lines.
STAT1 overexpression confers some tumours resistance against radiation and cisplatin treatment. ${ }^{32}$ Resistance against cisplatin and radiation based therapies is also frequent in MPM. Does increased STAT1 protein also induce resistance in MPM? Our data indicate that the axis STAT1-survivin can be associated with antiapoptotic and therapy resistant mechanisms in MPM, especially in patients with a poor prognosis, such as STS. Although similarly expressed in LTS, no similar association for STAT1 was found.

We also found high levels of MECP2 protein in LTS and STS. MECP2 particularly binds to methylated CpG islands in the genome and can be linked to DNA methylation and histone deacetylation. The implication of MECP2 in neoplasms has already been reported for a variety of human tumours. ${ }^{33}$ The prominent interaction between MECP2 and IGFR1 was detected in STS but not in LTS. At present, we are not able to explain the role of MECP2 in STS or in MPM generally. Interestingly, Gordon et al detected high levels of FMR1 mRNA (fragile $\mathrm{X}$ mental retardation 1), another X-linked gene, in MPM. ${ }^{34}$ The role of these X-linked genes, MECP2 and FMR1, in MPM is unknown, but involvement in the epigenetic silencing network is indicated.

Our study provides novel insights into the regulatory mechanisms of signalling pathways in MPM, which differentially promote tumour growth in LTS and STS. As shown, 
EGFR signalling is activated more strongly in LTS, whereas PDGFR signalling is more abundant in STS. Antiapoptosis is upregulated in STS by STAT1-survivin and related molecules, but not in LTS. We have demonstrated that small scale proteomics can be carried out by a powerful linkage of TMA, immunohistochemistry and statistical methods, to identify differences in protein expression. Nevertheless, these tools were used to generate a hypothesis. Additional in vitro studies are needed to evaluate multiple targeting strategies, including EGFR, PDGFR, TIE2, STAT1 and PCNA. This may provide new treatment options of these aggressive neoplasms. To the best of our knowledge, we have provided the first evidence of an immunohistochemistry based comparison with regard to signalling pathways in MPM between STS and LTS in a large series of LTS.

Acknowledgements: We gratefully thank Margit Gogg-Kamerer and Elisabeth Grygar, Institute of Pathology, Medical University of Graz, Austria, for technical support.

Funding: This work was supported by a research grant from the Austrian Cancer Aid/ Styria 04/2004 to HHP.

Competing interests: None.

Ethics approval: The study was approved by the local Ethical Commission.

\section{REFERENCES}

1. Ismail-Khan R, Robinson LA, Williams CC Jr, et al. Malignant pleural mesothelioma: a comprehensive review. Cancer Control 2006;13:255-63.

2. Robinson BW, Lake RA. Advances in malignant mesothelioma. $N$ Engl J Med 2005;353:1591-603.

3. Treasure T, Sedrakyan A. Pleural mesothelioma: little evidence, still time to do trials. Lancet 2004;364:1183-5.

4. Sugarbaker DJ, Flores RM, Jaklitsch MT, et al. Resection margins, extrapleural nodal status, and cell type determine postoperative long-term survival in trimodality therapy of malignant pleural mesothelioma: results in 183 patients. J Thorac Cardiovasc Surg 1999:117:54-63.

5. O'Kane SL, Cawkwell L, Campbell A, et al. Cyclooxygenase-2 expression predicts survival in malignant pleural mesothelioma. Eur J Cancer 2005;41:1645-8.

6. Parker C, Neville E. Lung cancer * 8: Management of malignant mesothelioma Thorax 2003;58:809-13.

7. Comin CE, Anichini C, Boddi V, et al. MIB-1 proliferation index correlates with survival in pleural malignant mesothelioma. Histopathology 2000;36:26-31.

8. Bongiovanni $\mathbf{M}$, Cassoni $\mathrm{P}$, De Giuli $\mathrm{P}$, et al. p27(kip1) immunoreactivity correlates with long-term survival in pleural malignant mesothelioma. Cancer 2001;92:1245-50.

9. Baldi A, Santini D, Vasaturo F, et al. Prognostic significance of cyclooxygenase-2 (COX-2) and expression of cell cycle inhibitors p21 and p27 in human pleural malignant mesothelioma. Thorax 2004;59:428-33.

10. Ramos-Nino ME, Vianale G, Sabo-Attwood T, et al. Cellular and molecular parameters of mesothelioma. J Cell Biochem 2006;98:723-34.

11. Whitson BA, Kratzke RA. Molecular pathways in malignant pleural mesothelioma. Cancer Lett 2006:239:183-9.
12. Jagadeeswaran R, Ma PC, Seiwert TY, et al. Functional analysis of c-Met/ hepatocyte growth factor pathway in malignant pleural mesothelioma. Cancer Res 2006;66:352-61.

13. Ordonez NG. What are the current best immunohistochemical markers for the diagnosis of epithelioid mesothelioma? A review and update. Hum Pathol 2007;38:1-16.

14. Ullmann R, Morbini P, Halbwedl I, et al. Protein expression profiles in adenocarcinomas and squamous cell carcinomas of the lung generated using tissue microarrays. J Pathol 2004;203:798-807.

15. Choi JK, Yu U, Yoo OJ, et al. Differential coexpression analysis using microarray data and its application to human cancer. Bioinformatics 2005;21:4348-55.

16. Mukohara T, Civiello G, Johnson BE et al. Therapeutic targeting of multiple signaling pathways in malignant pleural mesothelioma. Oncology 2005;68:500-10.

17. Ramos-Nino ME, Vianale G, Sabo-Attwood T, et al. Human mesothelioma cells exhibit tumor cell-specific differences in phosphatidylinositol 3-kinase/AKT activity that predict the efficacy of Onconase. Mol Cancer Ther 2005:4:835-42.

18. Zucali PA, Giaccone G. Biology and management of malignant pleural mesothelioma. Eur J Cancer 2006;42:2706-14.

19. Caja L, Ortiz C, Bertran E, et al. Differential intracellular signalling induced by TGFbeta in rat adult hepatocytes and hepatoma cells: Implications in liver carcinogenesis. Cell Signal 2007;19:683-94.

20. Ascoli V, Scalzo CC, Facciolo F, et al. Platelet-derived growth factor receptor immunoreactivity in mesothelioma and nonneoplastic mesothelial cells in serous effusions. Acta Cytol 1995;39:613-22.

21. Morelli PI, Martinsson S, Ostergren-Lunden G, et al. IFNgamma regulates PDGFreceptor alpha expression in macrophages, THP-1 cells, and arterial smooth muscle cells. Atherosclerosis 2006;184:39-47.

22. Buard A, Vivo C, Monnet I, et al. Human malignant mesothelioma cell growth: activation of janus kinase 2 and signal transducer and activator of transcription 1alpha for inhibition by interferon-gamma. Cancer Res 1998;58:840-7.

23. Shukla A, Gulumian M, Hei TK, et al. Multiple roles of oxidants in the pathogenesis of asbestos-induced diseases. Free Radic Biol Med 2003;34:1117-29.

24. Dales JP, Garcia S, Bonnier $\mathrm{P}$, et al. Tie2/Tek expression in breast carcinoma: correlations of immunohistochemical assays and long-term follow-up in a series of 909 patients. Int J Oncol 2003:22:391-7.

25. Takahama M, Tsutsumi M, Tsujiuchi T, et al. Enhanced expression of Tie2, its ligand angiopoietin-1, vascular endothelial growth factor, and CD31 in human non-small cell lung carcinomas. Clin Cancer Res 1999;5:2506-10.

26. Tanaka S, Mori M, Sakamoto Y, et al. Biologic significance of angiopoietin-2 expression in human hepatocellular carcinoma. J Clin Invest 1999;103:341-5.

27. Xia C, Xu Z, Yuan $X$, et al. Induction of apoptosis in mesothelioma cells by antisurvivin oligonucleotides. Mol Cancer Ther 2002;1:687-94.

28. Diaz N, Minton S, Cox C, et al. Activation of stat3 in primary tumors from high-risk breast cancer patients is associated with elevated levels of activated SRC and survivin expression. Clin Cancer Res 2006;12:20-8.

29. Fennell DA, Rudd RM. Defective core-apoptosis signalling in diffuse malignant pleural mesothelioma: opportunities for effective drug development. Lancet Oncol 2004;5:354-62.

30. Stephanou A, Latchman DS. Opposing actions of STAT-1 and STAT-3. Growth Factors 2005;23:177-82

31. Buettner R, Mora LB, Jove R. Activated STAT signaling in human tumors provides novel molecular targets for therapeutic intervention. Clin Cancer Res 2002:8:945-54.

32. Klampfer L. Signal transducers and activators of transcription (STATs): Novel targets of chemopreventive and chemotherapeutic drugs. Curr Cancer Drug Targets 2006; 6:107-21.

33. Prokhortchouk E, Hendrich B. Methyl-CpG binding proteins and cancer: are MeCpGs more important than MBDs? Oncogene 2002:21:5394-9.

34. Gordon GJ, Rockwell GN, Jensen R, et al. Identification of novel candidate oncogenes and tumor suppressors in malignant pleural mesothelioma using largescale transcriptional profiling. Am J Pathol 2005;166:1827-40. 